\begin{tabular}{|c|c|c|c|}
\hline Eiszeitalter und Gegenwart & $\mathbf{5 4}$ & $\begin{array}{l}36-47 \\
4 \mathrm{Abb} .\end{array}$ & Hannover 2004 \\
\hline
\end{tabular}

\title{
Das mittelpleistozäne Interglazial von Surheide
}

\author{
KARL-ERnSt BeHRE ${ }^{*}$
}

Keywords: Middle Pleistocene, interglacial, pollen analysis, NW Germany

Kurzfassung: In einer elster- bis saalezeitlichen Schichtenfolge in Surheide bei Bremerhaven wurde ein mittelpleistozänes limnisch ausgebildetes Interglazial angetroffen und pollenanalytisch untersucht. Der ältere und der mittlere Teil des Interglazials sind vorhanden, der jüngere Teil fehlt. Kennzeichnende Merkmale im Pollendiagramm sind ein früher und hoher Gipfel von Corylus und ein spätes Maximum von Abies sowie das völlige Fehlen von Carpinus, Ilex und Tertiärrelikten. Klimatisch erreichte das Interglazial etwa postglaziale Temperaturen. Im Vergleich mit anderen Pollendiagrammen scheiden Eem, Holstein s.l. und alle bekannten Cromer-Interglaziale aus. Große Ähnlichkeit besteht dagegen mit der unteren Warmzeit des in Südpolen weit verbreiteten Ferdinandow-Interglazials, das dort zwischen Elster I und Elster II gestellt wird. Nordisches Material unter dem Interglazial von Surheide spricht für eine entsprechende Einstufung auch dieses Interglazials.

\section{[A middle Pleistocene interglacial deposit at Surheide, N.W. Germany]}

Abstract: At Surheide, near Bremerhaven (NW Germany) an interglacial lacustrine deposit from a sequence of Elsterian to Saalian age has been pollen analytically investigated. The pollen diagram spans the lower and middle part of this interglacial while the upper part is missing. The main features of the pollen diagram include an early and substantial peak

"Anschrift des Verfassers: Prof. Dr. KarL-Ernst BeHre, Niedersächsisches Institut für historische Küstenforschung, Viktoriastraße 26/28, D-26382 Wilhelmshaven. E-mail: behre@nihk.de of Corylus, a late maximum of Abies as well as failure to record Carpinus, Ilex and Tertiary elements. It is estimated that temperatures comparable to those attained in the Holocene were attained. Comparison with other pollen diagrams shows that Eemian, Holsteinian s.l. and all known Cromerian interglacials can be excluded from consideration. There is, however, a strong similarity with the early warm period of the Ferdinandow interglacial, which is widely represented in southern Poland and further to the east, and is assumed to lie between the Elster I and Elster II phases. Material of nordic origin, recorded below the Surheide interglacial, also supports the view that this interglacial deposit may be assigned to this position in the glacial/interglacial sequence.

\section{Einleitung}

Bereits im Jahre 1971 wurde im Zuge des Baus der Autobahn 27 in dem zur Stadt Bremerhaven gehörenden Ort organisches Material angeschnitten. Es wurde von dem Heimatforscher R. EGGERS als wichtig erkannt, der auch den Autor informierte, sodass dieser die notwendigen Proben entnehmen konnte. Die später durchgeführten Pollenanalysen zeigten das Bild eines Interglazials, zu dem es keine Parallele gab. Aus diesem Grunde wurde die Publikation zunächst aufgeschoben, doch nachdem in der Zwischenzeit in Deutschland immer noch nichts Vergleichbares gefunden worden ist, wird das Material jetzt vorgelegt. 


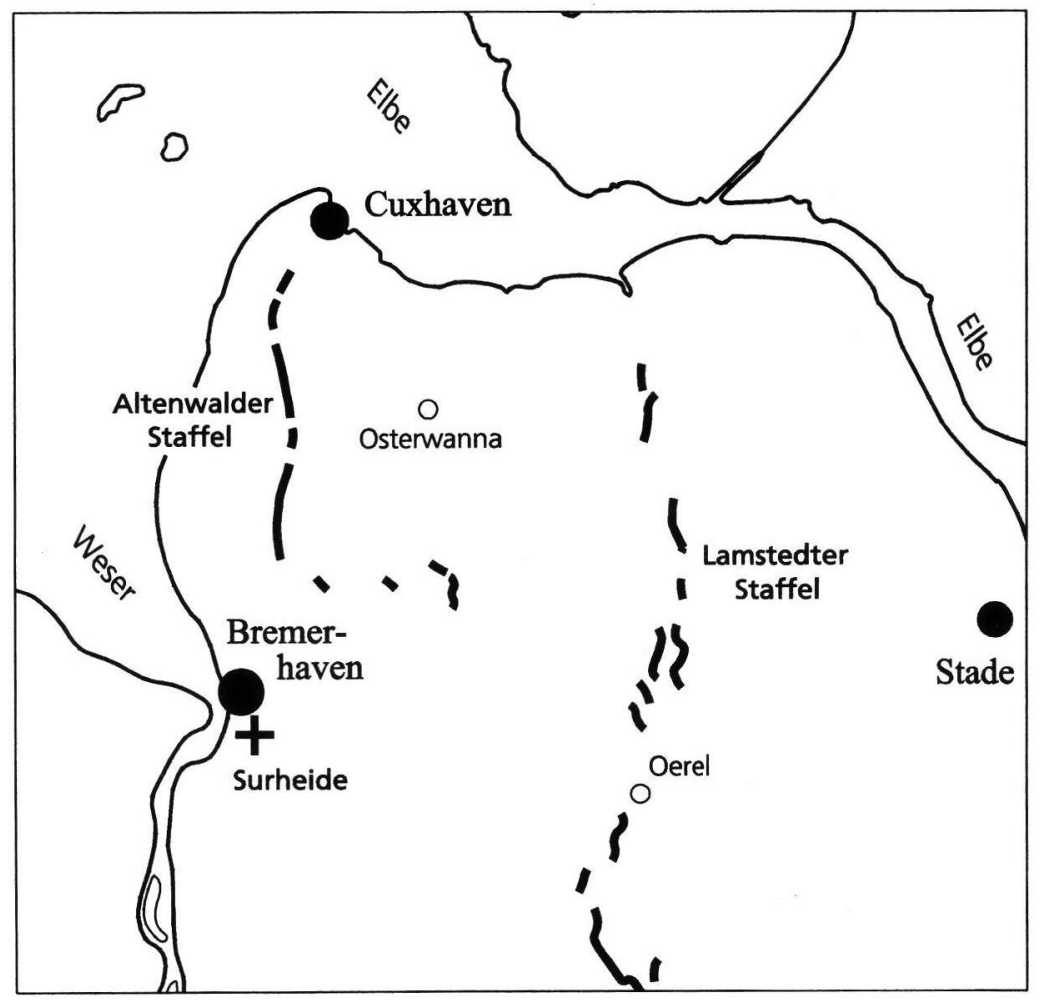

Abb. 1: Übersichtskarte des Elbe-Weser-Gebietes mit den Drenthe-II-zeitlichen Endmoränen der Altenwalder und Lamstedter Staffel

Fig. 1: General geological map of the Elbe-Weser region showing the location of the Drenthe-II end moraines Altenwalder and Lamstedter Staffel

\section{Untersuchungsraum und Geländebefunde}

Das Gebiet von Surheide (Abb. 1) liegt außerhalb der Drenthe-2-Endmoränen und wurde letztmalig während des Drenthe-1-Vorstoßes vom Eis überfahren. Oberflächlich besteht es aus Grundmoräne und Glazifluviatil und besaß an der Fundstelle eine ursprüngliche Höhe von $+7,5 \mathrm{~m} \mathrm{NN}$.

Der Fundplatz liegt bei Bremerhaven-Surheide auf der TK 25, Blatt 2417 Bremerhaven, bei den Koordinaten rechts 347542 und hoch 59 31 54. Dort führt die Brücke des Vieländerweges über die Autobahn und die organogene
Schicht wurde beim Ausheben des Fundamentlochs für den Mittelpfeiler angeschnitten. Die Fundbedingungen litten unter dem Zwang des schnellen Arbeitsfortschritts, sodass eingehende geologische Aufnahmen unterbleiben mussten. In der Baugrube war eine $5-30 \mathrm{~cm}$ mächtige organogene Schicht erkennbar, aus der innerhalb einer Breite von $5 \mathrm{~m}$ insgesamt 6 Profile für Pollenanalysen entnommen wurden. Von diesen wurden 4 Profile analysiert, zwei von diesen, SUR II und SUR V, werden in den Abbildungen 2 und 3 vorgelegt. Die organogene Schicht, vermutlich das Sediment eines ehemaligen Sees, hat sich in der Autobahntrasse 


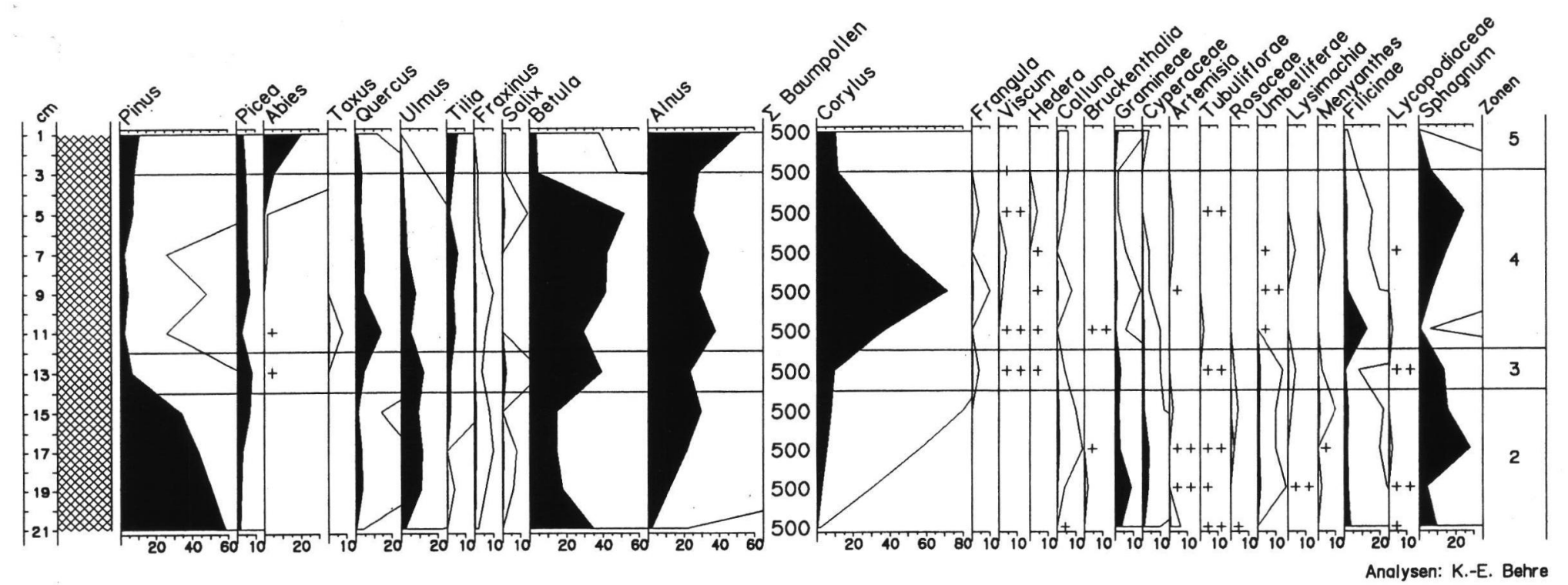

Abb. 2: Pollendiagramm Surheide II. Relativdiagramm mit Baumpollensumme als Berechnungsbasis.

Überhöhung =10:1.

Die folgenden Taxa wurden wegen sehr geringen Vorkommens nicht eingezeichnet (Anzahl u. Maximum des Vorkommens): Populus (1; 0,2), Acer (2; +), Vaccinium $(1 ; 0,2)$, Rumex acetosa-T. $(2 ; 0,2)$, Chenopodiaceae $(1 ; 0,2)$, Lychnis-T. $(1 ;+)$, Geranium $(1 ;+)$, Campanula-T. $(2 ; 0,6)$, Rubiaceae $(2 ; 0,2)$, Stellaria bolostea $(1 ;+)$, Ranunculaceae $(3 ; 0,2)$, Typha $(1 ;+)$, Polypodium vulgare $(1 ;+)$.

+ bedeutet einmal, ++ mehrfach außerhalb der Zählung

Fig. 2: Pollen diagram Surheide II. Taxa in percentage of AP. Exaggeration = 10:1. The taxa mentioned above have not been included because of scarce occurrence (in brackets number and maximum of occurrence):

+ means once, ++ several times outside the counting 


\section{Surheide $V$}

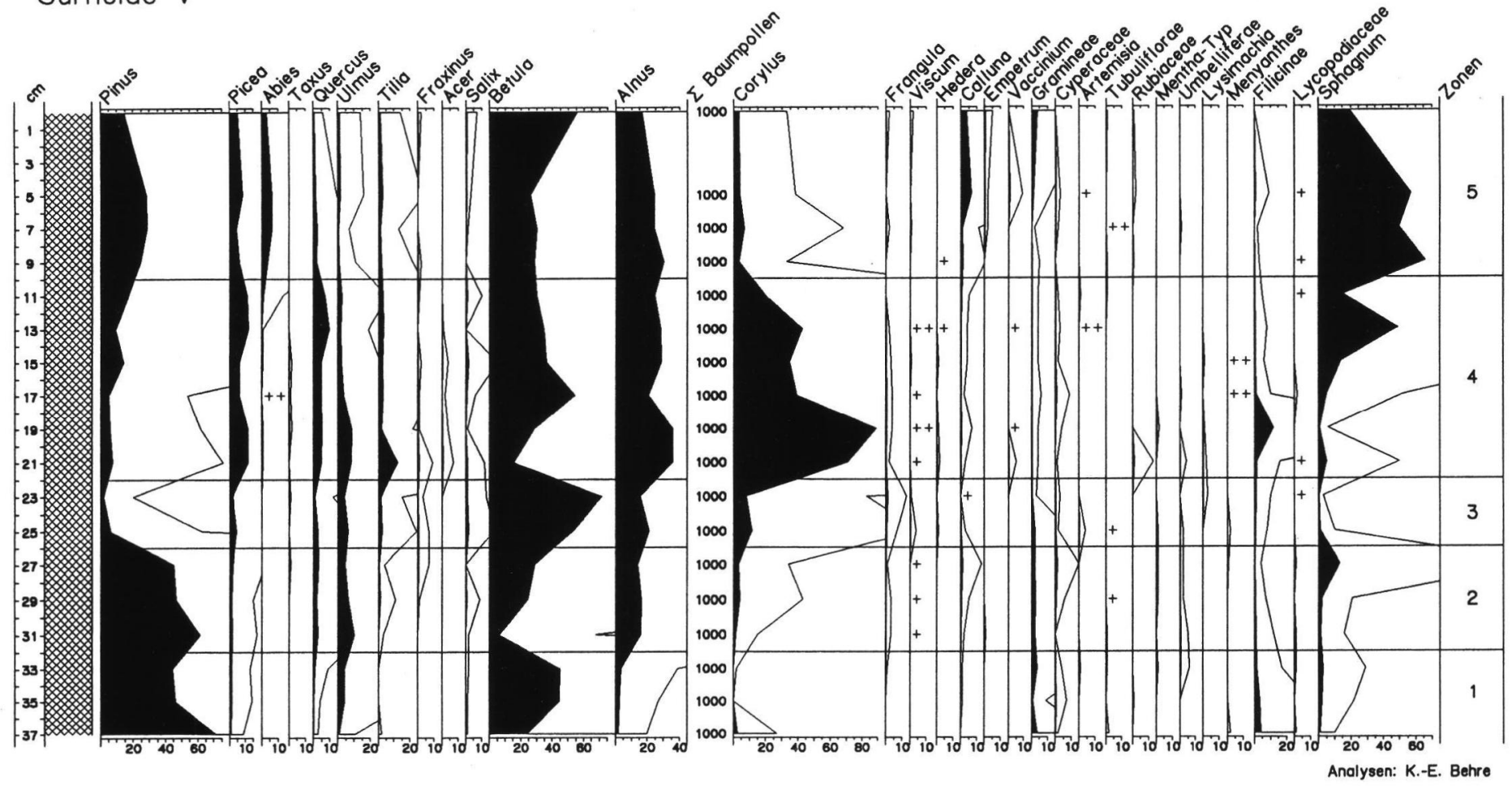

Abb. 3: Pollendiagramm Surheide V. Relativdiagramm mit Baumpollensumme als Berechnungsbasis. Überhöhung = 10:1.

Die folgenden Taxa wurden wegen sehr geringen Vorkommens nicht eingezeichnet (Anzahl u. Maximum des Vorkommens): Fagus (1; +), Humulus-T. $(1 ;+)$, Viburnum-T. $(1 ; 0,1)$, Campanula-T. $(1 ; 0,1)$, Jasione-T. $(1 ; 0,1)$, Caryophyllaceae $(2 ; 0,1)$, Liguliflorae $(1 ;+)$, Chenopodiaceae $(4 ; 0,1)$, Epilobium $(1 ;+)$, Potentilla-T. (2;0,1), Filipendula $(1 ; 0,5)$, Anemone-T. $(1 ; 0,1)$, Thalictrum $(2 ; 0,2)$, Typha $(3 ; 0,4)$, Sparganium-T. $(3 ; 0,2)$, Nymphaea $(1 ; 0,1)$, Utricularia $(1$; $0,1)$, Polypodium vulgare $(5 ;++)$, Osmunda $(1 ; 0,1)$, Ophioglossum $(1 ; 0,1)$.

+ bedeutet einmal, ++ mehrfach außerhalb der Zählung

Fig. 3: Pollen diagram Surheide V. Taxa in percentage of AP. Exaggeration = 10:1.

The taxa mentioned above have not been included because of scarce occurrence (in brackets number and maximum of occurrence):

+ means once, ++ several times outside the counting 
noch erheblich weiter nach Norden fortgesetzt, konnte vom Autor selber aber nicht mehr beobachtet werden.

Die Stratigraphie sah an der Fundstelle wie folgt aus:

\begin{tabular}{|c|c|}
\hline $0-100 \mathrm{~cm}$ & Auftragsboden \\
\hline $100-400 \mathrm{~cm}$ & glaziofluviatiler Sand in \\
\hline $400-520 \mathrm{~cm}$ & $\begin{array}{l}\text { geschichteter Mittel- und } \\
\text { Grobsand, z.T. mit Steinen } \\
\text { (Flint etc.) („Vorschüttsande“) }\end{array}$ \\
\hline $520-573 \mathrm{~cm}$ & $\begin{array}{l}\text { toniger Feinsand mit } \\
\text { zahlreichen Eisenoxidbändern }\end{array}$ \\
\hline $573-644 \mathrm{~cm}$ & toniger Feinsand \\
\hline $644-714 \mathrm{~cm}$ & Fein- und Mittelsand \\
\hline $714-732 \mathrm{~cm}$ & $\begin{array}{l}\text { Torf bzw. organische Mudde } \\
\text { (Interglazial) }\end{array}$ \\
\hline $\mathrm{ab} 732 \mathrm{~cm}$ & $\begin{array}{l}\text { gemischte Sande mit groben } \\
\text { Steinen (Grundmoräne?) } \\
\text { darunter heller Feinsand }\end{array}$ \\
\hline
\end{tabular}

Entscheidendes Merkmal des Profils sind die z.T. mehrere $\mathrm{cm}$ großen abgerundeten Gesteine unterhalb des Interglazials. Dabei handelt es sich um stark verwitterten Flint und nordisches Kristallin, ein Befund, der dankenswerterweise von Prof. Dr. K.-D. Meyer, Hannover, bestätigt wurde. Ebenfalls K.-D. Meyer teilte dem Autor Archivbohrungen des näheren Umfeldes aus dem Niedersächsischen Landesamt für Bodenforschung mit, die sicher über $20 \mathrm{~m}$ Pleistozänmächtigkeit aufweisen.

Nach dem stratigraphischen Befund kommt für das Interglazial sowohl das Holstein in Frage als auch ein intra-Saale- oder intra-Elster-zeitliches Alter. Eine aufgeschuppte ältere Scholle ist unwahrscheinlich, da eine solche verbogen sein oder zumindest schräg liegen sollte, dagegen ist die organogene Schicht über die ganze Länge horizontal gelagert und hat keine erkennbaren Störungen.

\section{Die Pollendiagramme}

\section{Das Material}

Die organogene Schicht war nach oben und unten scharf begrenzt. Gelegentlich traten stark gepresste dünne Holzreste auf, die als Pinus (6 $\mathrm{x})$, Betula $(1 \mathrm{x})$ und Salix $(1 \mathrm{x})$ bestimmt wurden. Darüber hinaus wurden beim Aufbrechen des organischen Materials einige Früchtchen und Samen gesammelt. Dabei handelt es sich um mehrere Bruchstücke von cf. Pinus-Samen, dazu um 17 Früchtchen von Potamogeton spec., 4 Früchtchen von Ceratophyllum demersum, 4 von Carex spec. und einen Samen von Menyanthes trifoliata. Danach muss an dieser Stelle zumindest zeitweise ein See bestanden haben.

Die Pollenprofile wurden in Zinkkästen entnommen und die Proben in der üblichen Weise mit Azetolyse aufbereitet. Dabei stellte sich heraus, daß das organogene Material fast ausschließlich aus reiner Pollenmasse bestand. Derartige Bildungen wurden bereits früher aus dem Holozän als Pollengyttja oder Fimmenit beschrieben und als im Wasser zusammengeschwemmte Pollenmassen erklärt (Overbeck 1975: 87). In Surheide dürfte eine anaerobe Zersetzung hinzugekommen sein, der die resistenteren Sporopollenine der Pollenwände besser widerstanden als Gewebereste.

Die Pollenerhaltung war durchweg gut, die Proben wurden bei einem Abstand von $2 \mathrm{~cm}$ im Profil SUR II auf 500 und im Profil SUR V auf 1000 Baumpollen ausgezählt, sodass eine hohe statistische Sicherheit gewährleistet ist.

Lokale Verhältnisse und Ablagerungsbedingungen

Aus den Pollenanalysen geht hervor, dass das Gebiet von Surheide während des gesamten erfassten Zeitabschnitts von dichtem Wald bedeckt war. Im strauchigen Unterwuchs domi- 
nierte Corylus, begleitet von etwas Frangula alnus. Zwergsträucher gab es kaum. Sie bestanden vor allem aus Calluna, daneben aus Empetrum und Vaccinium sowie vereinzelt Bruckenthalia und blieben mit Ausnahme der obersten drei Proben von SUR V zusammen stets unter $1 \%$. Der Krautpollen blieb bis auf den unteren Teil von SUR II, wo er maximal 15,6\% erreicht, unter 5 $\%$; das meiste davon waren Gramineen.

Nimmt man als Klimaindikatoren Tilia, Viscum und Hedera, dann entspricht das damalige Klima dem der Nacheiszeit. Vegetationsabweichungen zu anderen klimatisch ähnlichen Interglazialvorkommen müssen deshalb vor allem auf unterschiedliche Einwanderungsbedingungen, daneben auf andere Standortverhältnisse zurückzuführen sein.

Bereits die schon genannten Makroreste von Potamogeton und Ceratophyllum demersum weisen auf limnische Verhältnisse, dieses wird unterstützt durch einzelne Pollenkörner von Nymphaea und Utricularia.

An charakteristischen Sumpfpflanzen treten in den Pollenanalysen neben Cyperaceae (dazu 4 Carex-Früchtchen) vor allem regelmäßig $M e$ nyanthes, daneben Lysimachia, wenig Typha angustifolia und latifolia sowie vereinzelt Thalictrum und Filipendula auf. Hinzu kommen z.T. erhebliche Werte von Sphagnum-Sporen.

Danach repräsentiert die Fundstelle einen See, umgeben von einem versumpften Rand. Utricularia, Menyanthes und Sphagnum weisen dabei auf oligo- bis mesotrophe Verhältnisse hin.

\section{Der Vegetationsablauf}

Trotz der geringen Mächtigkeit des interglazialen Torfs von maximal $37 \mathrm{~cm}$ ist ein wesentlicher Teil der Warmzeit erfasst, der in den Pollendiagrammen mit Probenabständen von 2 bzw. 2,5 cm entsprechend aufgelöst wurde.

Die Diagramme beginnen in der lokalen Pollenzone 1 mit einer Dominanz von Pinus und
Betula, die auf den Beginn des Interglazials hinweisen. Thermophile Bäume, wie Quercus, Ulmus und Alnus, sind zwar schon zum Teil anwesend, jedoch erst in geringen Werten.

Mit dem Beginn der geschlossenen Tilia-Kurve und dem Anstieg von Alnus setzt die Pollenzone 2 ein, die bis zum endgültigen Niedergang von Pinus reicht. Der nun in Pollenzone 3 folgende Betula-Anstieg hat sicher keine klimatische Bedeutung, denn die thermophilen Laubbäume zeigen keine Reaktion; die Ursache ist wahrscheinlich eine lokale Vernässung, die sich in den Kurven von Lysimachia und Menyanthes zeigt und die länger anhält.

Sehr markant ist die Phase der hohen CorylusWerte, deren Maximum 89,3\% (bezogen auf Baumpollen) beträgt und die Pollenzone 4 bildet. Nach Ausweis der Eichenmischwaldarten, vor allem von Tilia, ist jetzt das klimatische Optimum erreicht.

In der abschließenden Pollenzone 5 gehen Betula sowie der Eichenmischwald zurück, gleichzeitig breitet sich jetzt Abies aus, die hier bis zu $21 \%$ erreicht. Auf den trockenen Böden dominieren nun die Nadelhölzer mit Abies, Picea und jetzt auch wieder Pinus.

Kennzeichnend gegenüber anderen Interglazialdiagrammen sind für Surheide der hohe Corylus-Gipfel, das frühe Auftreten von Picea (vor Corylus), der Abies-Gipfel am Schluss und dazu das vollständige Fehlen von wichtigen Arten, wie Carpinus und Ilex sowie von Arolla und allen anderen Tertiärrelikten - und das bei einer Auszählung auf 1000 Baumpollen/Probe in Surheide V.

\section{Zur Einordnung von Surheide in die Quartärstratigraphie}

Ohne Berücksichtigung der stratigraphischen Gegebenheiten muss zunächst geprüft werden, wo sich die Fundschicht nach rein palynologischen Kriterien einordnen lässt. Dazu können 


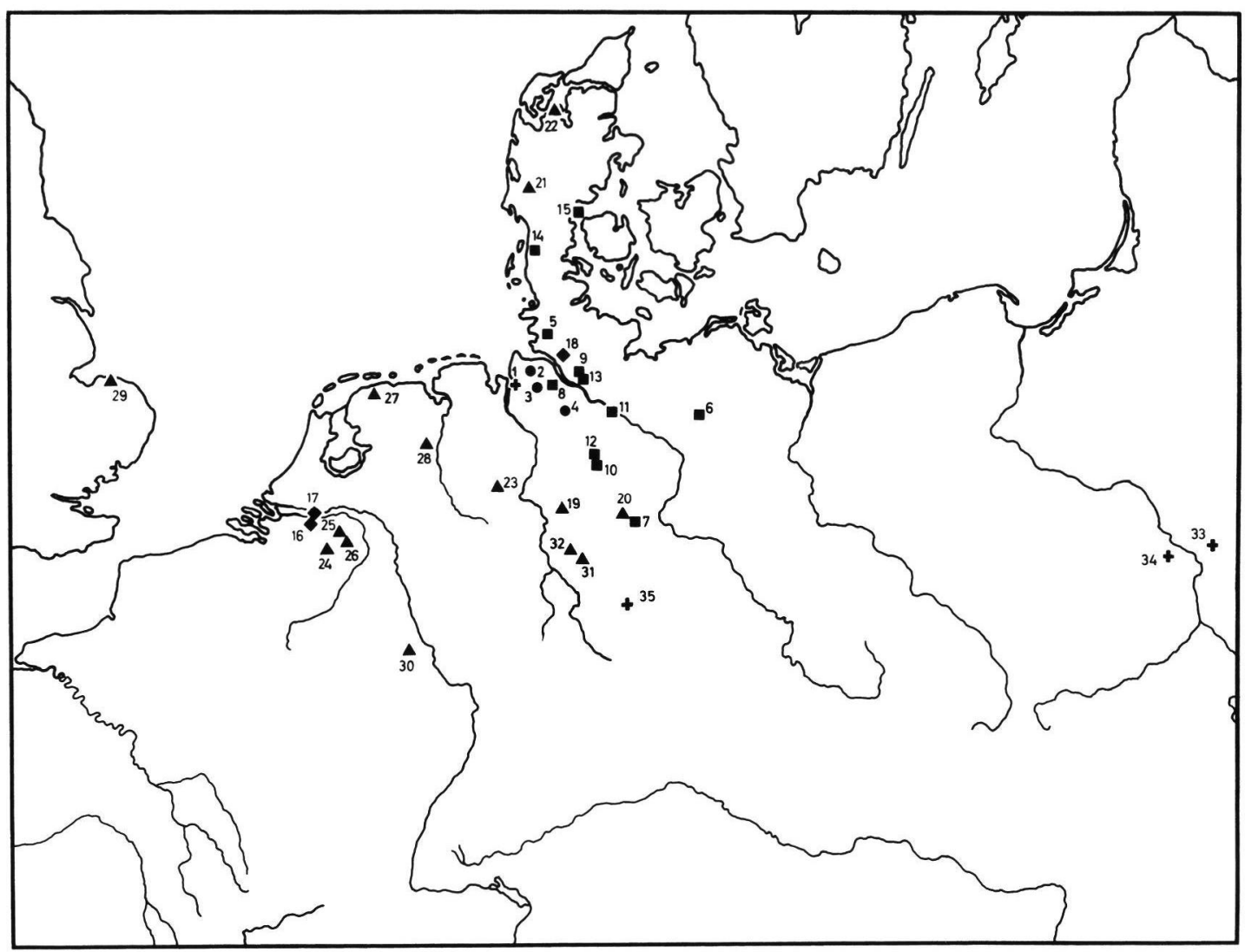

Eem

Holstein s.I.

Cromer

Altpleistozän $\boldsymbol{+}$ andere Interglaziale

Abb. 4: Die Lage der im Text erwähnten Profilstellen

Fig. 4: Location of the sites mentioned in the text

\begin{tabular}{|c|c|c|c|c|c|}
\hline 1 & Surheide & 16 & Bavel/NL & 31 & Bilshausen \\
\hline 2 & Osterwanna & 17 & Leerdam/NL & 32 & Göttingen \\
\hline 3 & Oerel & 18 & Lieth & 33 & Ferdinandów/PL \\
\hline 4 & Gr.Todtshorn & 19 & Osterholz & 34 & Stanislawice/PL \\
\hline 5 & Wacken & 20 & Elm & 35 & Voigtstedt \\
\hline 6 & Pritzwalk & 21 & Olgød/DK & & \\
\hline 7 & Schöningen & 22 & Harreskov/DK & & \\
\hline 8 & Bossel & 23 & Hunteburg & & \\
\hline 9 & Hamburg-Hummelsbüttel & 24 & Westerhoven & & \\
\hline 10 & Wiechel & 25 & Rosmalen/NL & & \\
\hline 11 & Breetze & 26 & Het Zwinkel/NL & & \\
\hline 12 & Munster & 27 & Noordbergum/NL & & \\
\hline 13 & Hamburg-Dockenhuden & 28 & Roswinkel/NL & & \\
\hline 14 & Tornskov/DK & 29 & Cromer/GB & & \\
\hline 15 & Vejlby/DK & 30 & Kärlich & & \\
\hline
\end{tabular}


einige in den letzten Jahren vorgelegte Pollendiagramme aus Norddeutschland herangezogen werden.

Der hohe Corylus-Gipfel erinnert an ähnliches Verhalten im Eem, von dem aus der engeren Nachbarschaft u.a. die Diagramme Osterwanna $^{2^{*}}$ (Behre 1974), Oerel $^{3}$ (Behre \& Lade 1986) und Groß Todtshorn ${ }^{4}$ (CASPERs 1997) vorliegen (vgl. Abb. 1). Danach müsste jedoch Carpinus in geringen Werten bereits ab dem Corylus-Abfall erscheinen und bald zunehmen, Taxus im oberen Bereich von Surheide 1 über 10 $\%$ erreichen, Abies sehr viel später als in Surheide kommen, nämlich gleichzeitig mit dem hier nicht vorhandenen Carpinus-Abfall, und auch Ilex müsste vom Corylus-Gipfel an regelmäßig vorkommen. Allein anhand dieser Kriterien lässt sich ein eemzeitliches Alter für Surheide mit Sicherheit ausschließen.

Eine Einordnung des Surheide-Vorkommens in das Holstein-Interglazial bzw. das folgende Wacken-Dömnitz-Interglazial muss ebenfalls geprüft werden. Hier liegen zum Vergleich die Profile aus Wacken ${ }^{5}$ (Menke 1968), Pritzwalk ${ }^{6}$ (ERd 1973) und Schöningen ${ }^{7}$ (Urban et al. 1991) mit beiden Interglazialen vor, sowie darüber hinaus die benachbarten Profile Bossel ${ }^{8}$ (Müller \& HöFle 1994), Hamburg-Hummelsbüttel ${ }^{9}$ und Wiechel ${ }^{10}$ (Hallik 1960), von Breetze/Elbe ${ }^{11}$ (Benda \& Meyer 1973), Munster $^{12}$ (MüLler 1974) und Dockenhuden ${ }^{13}$ (Linke \& Hallik 1993) sowie die aus Tornskov $^{14}$ und Vejlby ${ }^{15}$ in Dänemark (ANDERSEN 1965). Allen gemeinsam ist das regelmäßige Vorkommen von Carpinus und Ilex, die in Surheide beide trotz sehr hoher Auszählung fehlen. Allein dieses schließt ein holsteinzeitliches Alter aus. Hinzu kommen weitere Merkmale, wie die Corylus-Kurve, die nur in Munster einmal $30 \%$ erreicht, in allen anderen Pollendiagrammen jedoch unter $20 \%$ bleibt - gegenüber bis 89,3 $\%$ in Surheide. Taxus, die in Surheide nur sporadisch mit einzelnen Pollenkörnern auftritt, ist im Holstein und Wacken eine voll etablierte
Art und erreicht während der haselreicheren Zeit in Munster und Vejlby Werte an die $20 \%$. Die genannten Charakteristika zeigen, dass die Profile von Surheide mit Sicherheit nicht in den Holstein-Komplex gehören.

Die altpleistozänen Interglaziale scheiden vor allem wegen der dortigen Tertiärelemente ebenfalls aus. Die jüngsten Interglaziale dieses Abschnittes, in den Niederlanden Bavel ${ }^{16}$ und Leerdam $^{17}$, haben nach ZAGWIJN \& DE JONG 1984 immer noch Exoten, vor allem Eucommia (und das Bavel auch viel Tsuga), außerdem führen sie durchgehend Carpinus und Ilex bei Corylus-Werten unter $10 \%$. Die entsprechenden Interglaziale in der Lieth-Serie ${ }^{18}$ aus Schleswig-Holstein, Ütersen und Pinneberg (MenKe 1975) sind mit hohen Ericales-Werten stark lokal geprägt. Ihnen fehlen jedoch Abies völlig, Tilia und Corylus fast ganz, dafür haben sie fast durchgehend Carpinus und Larix sowie geringe Werte verschiedener Tertiärrelikte. Nach den genannten Merkmalen lassen sowohl die älteren als auch die jüngeren altpleistozänen Interglaziale eine Einordnung von Surheide nicht zu.

Noch immer sehr lückenhaft sind unsere Kenntnisse über die Interglaziale des Cromer-Komplexes, von denen nicht einmal die genaue Zahl feststeht. Für die Niederlande hat ZAGWIJN (1975) Cromer-Interglaziale I bis IV beschrieben, mit denen in den benachbarten Ländern eine Korrelation versucht wird. Nach dem Ausschluß der bekannten älteren und jüngeren Interglaziale muss für Surheide eine Einordnung in das Cromer besonders intensiv geprüft werden.

Das Cromer I, für das vor allem die langen Profile Osterholz ${ }^{19}$ und Elm ${ }^{20}$ (GRÜGER 1968) repräsentativ sind, scheidet wegen der hohen Eichenmischwaldwerte, dem Vorkommen von Carpinus und der nur sporadischen Abies-Funde aus.

Gewisse Ähnlichkeiten bestehen dagegen mit dem Cromer II, in das die Vorkommen von Ol$\mathrm{g} ø^{21}$ und Harreskov ${ }^{22}$ in Dänemark (ANDER- 
SEN 1965), Hunteburg ${ }^{23}$ (HAHNe 1994) und Westerhoven ${ }^{24}$ (ZAGWIJN \& ZonNeveld 1956) gestellt werden, wobei vor allem der CorylusGipfel und das Verhalten von Picea vergleichbar sind. Dagegen müssten die frühen und sehr hohen Werte von Taxus und Ulmus sich auch in Surheide niederschlagen und abgesehen von Dänemark kommt auch Carpinus vor. Auch dieses Interglazial fällt somit für Surheide aus. Das aus Rosmalen ${ }^{25}$ und Het Zwinkel ${ }^{26}$ (ZAGwIJN, zuletzt 1996) beschriebene Cromer III ist noch sehr unvollständig, enthält aber sowohl Carpinus als auch Ilex und kommt damit nicht in Frage.

Auch das aus Noordbergum ${ }^{27}$ beschriebene, noch sehr unvollständige Cromer IV (ZAGWIJN 1996), in das auch Roswinkel ${ }^{28}$ und das klassische obere Cromer Forest Bed ${ }^{29}$ (West Runton, WEST 1980) gestellt werden, entfällt wegen der nur geringen Werte von Corylus und Abies und dem Vorkommen von Carpinus.

In ein jüngeres Cromer-Interglazial werden die vergleichsweise vollständigen Vorkommen von Kärlich ${ }^{30}$ und Bilshausen ${ }^{31}$ gestellt (BitTMANN \& Müller 1996). Mit einem hohen CorylusGipfel zu Beginn haben sie deutliche Ähnlichkeit mit Surheide, andererseits schließen jedoch hohe Werte von Carpinus und das Auftreten von Ilex eine Korrelation mit unserem Interglazial aus.

Aus diesen Darlegungen wird erkennbar, dass die Zuordnung des Interglazials Surheide zu einem in Mitteleuropa bekannten CromerInterglazial nicht möglich ist. Das gleiche gilt im übrigen auch für drei übereinanderliegende Warmzeiten, die GrüGer et al. (1994) aus Göttingen-Ottostraße ${ }^{32}$ publizierten, die aber wiederum von Surheide abweichen.

Geht man über den engeren mitteleuropäischen Bereich hinaus, dann trifft man in Südpolen auf das Ferdinandow-Interglazial ${ }^{33}$ (JАNCYK-KoPIKOWA 1975), das inzwischen an 9 weiteren südpolnischen Stellen nachgewiesen wurde (Rzechowski 1996), auch in Weißrussland und Russland ist es bekannt. Dieses Interglazial besteht aus zwei Warmzeiten, unterbrochen von einer längeren Betula-Pinus-Phase. Die untere der beiden Warmzeiten, gut erfasst auch in Stanislawice ${ }^{34}$, JANCYK-KopIKOWA \& ZARSKI 1995, weist große Ähnlichkeiten mit dem Pollendiagramm Surheide auf: hohe Corylus-Werte, vor denen wie in Surheide schon Ulmus und Quercus erscheinen, allerdings mit maximal etwa $20 \%$, d.h. höher als bei uns. Almus (früh), Tilia (später und gering), Taxus (sehr wenig), Acer und Fraxinus verhalten sich ebenfalls sehr ähnlich wie in Surheide, Picea hat einen ähnlichen Kurvenverlauf, ist aber schwächer. Von besonderem Gewicht sind der späte $A$ Abies-Gipfel und vor allem das völlige Fehlen von Carpinus, das in Polen als wesentlichstes Merkmal dieses Interglazials herausgestellt wird.

Abweichend von Surheide ist in Ferdinandow das zwar geringe, jedoch mehr oder weniger regelmäßige Auftreten von Ilex, Celtis, Vitis, Syringa und Ligustrum. Abgesehen von Ilex haben diese Arten eine vorwiegend südlich-kontinentale Verbreitung, sodass ihr Fehlen in Surheide, wo sie auch heute nicht natürlich vorkommen würden, verständlich ist.

Insgesamt ist die Übereinstimmung der Pollendiagramme Surheide und Ferdinandow so groß, dass man die Vorkommen wohl als gleichalt ansehen darf. Entscheidend ist nicht nur das ähnliche Bild der Haupt-Waldbildner, sondern vor allem die gleiche Einwanderungsund Ausbreitungsfolge dieser Arten. Carpinus hat es in diesem Interglazial möglicherweise überhaupt nicht geschafft, nach Mitteleuropa zurückzukehren. - Bei der weiten Entfernung von rd. $900 \mathrm{~km}$ zwischen Nordwestdeutschland und Südpolen, dazu noch in der Richtung atlantisch-kontinental, könnte man noch erheblich größere als die genannten Abweichungen erwarten.

Das Ferdinandow-Interglazial wurde bereits 1978 von ERD mit der Artern-Warmzeit von Voigtsted ${ }^{35}$ in Thüringen parallelisiert. Auch 
zwischen Artern und Surheide gibt es zahlreiche Übereinstimmungen, die eine Korrelierung durchaus möglich machen würden. Allerdings wurde in Voigtstedt der jüngere Teil der ArternWarmzeit ohne einen Corylus-Gipfel erfasst, während in Surheide der jüngste Teil fehlt, dafür im älteren Teil der hohe Corylus-Gipfel bestimmend ist. Die Parallelisierung beider Warmzeiten ist nicht zwingend, auch wenn das wichtige Merkmal des völligen Fehlens von Carpinus in beiden Profilen augenfällig ist. In Voigtstedt bildet das Interglazial die Basis der dort nur einfach ausgebildeten Elster-Grundmoräne (MEYER 1981).

\section{Diskussion}

Mehrere gute Merkmale, wie besonders ein hoher Corylus-Gipfel, ein spätes Maximum von Abies sowie das Fehlen von Carpinus, Ilex und Azolla kennzeichnen das Interglazial von Surheide so gut, dass es sich klar von anderen Vorkommen unterscheidet. Der Vergleich mit allen wichtigen Pollendiagrammen Mitteleuropas vom späten Altquartär bis zum Eem erbrachte keine Übereinstimmung mit einem bekannten Interglazial dieses Raumes. Dagegen bestehen trotz der weiten Entfernung große Ähnlichkeiten mit der unteren Warmzeit des in Südpolen mehrfach vertretenen Ferdinandow-Interglazials und seinem Äquivalent in Weißrussland und Russland (vgl. zum letzteren Rzechowski 1996, ZAGWIJN 1996).

Stratigraphisch wird das Ferdinandow-Interglazial in Polen heute von den meisten Autoren zwischen die Moränen von San 1 und San 2 gestellt (Mojski 1995, Lindner 1995, RZECHOWSKI 1996), doch gelegentlich wird auch eine Einordnung oberhalb von San 2, aber vor dem Masovian (Holstein) versucht (KrZyszKowski 1991). San 1 und 2 entsprechen im mitteldeutschen Raum Elster I und II (EIssmann 1995), die damit durch ein richtiges Interglazial getrennt wären. In Nordwestdeutschland wird in der Regel nur eine Elstermoräne kartiert, doch gibt es gelegentlich auch hier Hinweise auf zwei Vorstöße mit zwei Grundmoränen, wie im Elbe-Weser-Gebiet auf Blatt Buxtehude (Meyer 1982) und im Kreis Osterholz (Höfle 1983).

Für die Fundstelle Surheide reichen die bei der Notbergung des Profils gemachten Aufzeichnungen nicht für eine genauere stratigraphische Einstufung aus. Sicher ist die Position unterhalb oder innerhalb der Saale-Kaltzeit. Wegen des unmittelbar unter der organogenen Mudde liegenden nordischen Materials wäre ein präElster-Alter kaum zu erklären, es sei denn, man nimmt eine vorangegangene weitere Vereisung an, wofür es hier bislang keine Anzeichen gibt. $\mathrm{Da}$ nach den pollenanalytischen Befunden Holstein und Jüngeres ausscheiden, liegt nahe, das Profil Surheide in den Elsterbereich einzuordnen. Dieses wird durch die Korrelation mit dem wahrscheinlich intra-elsterzeitlichen Ferdinandow-Interglazial nachdrücklich gestützt. Die fehlende Übereinstimmung mit den bis jetzt bekannten Cromer-Interglazialen spricht ebenso dafür wie der völlige Mangel an Tertiärrelikten. Dabei kann allerdings das Ausbleiben von Azolla möglicherweise auf der Nährstoffarmut des Sees beruhen.

Zusammen mit einigen angeführten anderen neuen Pollendiagrammen zeigt das Profil Surheide, dass die genaue Zahl der Warmzeiten im Mittelpleistozän noch nicht bekannt und damit die Gliederung dieser Zeitperiode noch immer sehr unsicher ist.

\section{Dank}

Herr R. Eggers wies den Autor auf die Fundstelle hin und gab wesentliche Geländeunterstützung; Prof. Dr. E. Grüger und Prof. Dr. K.-D. Meyer sahen das Manuskript kritisch durch, die technischen Arbeiten führten Frau K. Petzel, 
K. Lehners und M. Spohr durch und Frau M. Janssen schrieb den Text. Ihnen allen gilt mein Dank.

\section{Literatur}

Andersen, S.T. (1965): Interglacialer og interstadialer i Danmarks kvartaer. - Medd. dansk geol. Foren., 15 (4): 486-506, 9 Abb.; Kopenhagen.

Behre, K.-E. (1974): Die Vegetation im Spätpleistozän von Osterwanna/ Niedersachsen. - Geol. Jb., A 18: 3-48, 8 Abb., 5 Taf.; Hannover.

Behre, K.-E. \& Lade, U. (1986): Eine Folge von Eem und 4 WeichselInterstadialen in Oerel/Niedersachsen und ihr Vegetationsablauf. - Eiszeitalter u. Gegenwart, 36: 11-36, 12 Abb., 2 Tab., 2 Taf.; Hannover.

Benda, L. \& Meyer, K.-D. (1973): Das Holstein-Interglazial von Breetze bei Bleckede/Elbe. - Geol. Jb., A 9: 21-40, 3 Abb., 2 Tab.; Hannover.

Bittmann, F. \& Müller, H. (1996): The Kärlich Interglacial site and its correlation with the Bilshausen sequence. In: Turner, Ch. [ed.]: The Early Middle Pleistocene in Europe, 187-193; Rotterdam, (Balkema).

Caspers, G. (1997): Die eem- und weichselzeitliche Hohlform von Groß Todtshorn (Kr. Harburg; Niedersachsen). Geologische und palynologische Untersuchungen $\mathrm{zu}$ Vegetation und Klimaverlauf der letzten Kaltzeit. Schriftenr. Dt. Geol. Ges., 4: 7-59, 38 Abb., 6 Tab.; Hannover.

Eissmann, L. (1995): Sachsen. In: Benda, L. [Hrsg.]: Das Quartär Deutschlands: 171-198, 7 Abb., 1 Tab.; Stuttgart (Borntraeger).

ERD, K. (1973): Pollenanalytische Gliederung des Pleistozäns der Deutschen Demokratischen Republik. - Z. geol. Wiss.,
1 (9): 1087-1103, 8 Abb., 1 Tab.; Berlin. ErD, K. (1978): Pollenstratigraphie im Gebiet der skandinavischen Vereisungen. - Schr.-R. geol. Wiss., 9: 99-119, 3 Abb.; Berlin.

GRÜGER, E. (1968): Vegetationsgeschichtlic he Untersuchungen an cromerzeitlichen Ablagerungen im nördlichen Randgebiet der deutschen Mittelgebirge. - Eiszeitalter u. Gegenwart, 18: 204-235, 3 Abb., 1 Tab., 5 Taf.; Öhringen/Württ.

Grüger, E., Jordan, H., Meischner, D. \& Schlie, P. (1994): Mittelpleistozäne Warmzeiten in Göttingen, Bohrungen Ottostraße und Akazienweg. - Geol. Jb., A 134: 167-209, 6 Abb., 4 Tab., 1 Taf.; Hannover.

Hahne, J., Mengeling, H., Merkt, J. \& Gramann, F., (1994): Die HunteburgWarmzeit („Cromer-Komplex“) und Ablagerungen der Elster-, Saale- und Weichsel-Kaltzeit in der Forschungsbohrung Hunteburg GE 58 bei Osnabrück. - Geol. Jb., A 134: 117-165, 21 Abb., 4 Tab.; Hannover.

Hallik, R. (1960): Die Vegetationsentwicklung der Holstein-Warmzeit in Nordwestdeutschland und die Altersstellung der Kieselgurlager der südlichen Lüneburger Heide. - Z. dt. geol. Ges., 112: 326-333, 3 Abb.; Hannover.

Höfle, H.-Chr. (1983): Strukturmessungen und Geschiebeanalysen an eiszeitlichen Ablagerungen auf der Osterholz-Scharmbecker Geest. - Abh. naturwiss. Ver. Bremen, 40: 39-53, 11 Abb., 2 Tab.; Bremen.

JaNCYK-Kopikowa,Z. (1975): Florainterglacjalu Marzowieckiego w Ferdinandowie.- Biuletyn Institut Geologiczny, 290: 1-94; Warschau. JanCYK-Kopikowa, Z. \& ZarsKi, M. (1995): The Ferdinandów interglacial at Stanislawice near Kozienice (Central Poland). - Acta Palaeobotanica, 35, 7-13; Warschau u. Krakau.

Krzyszkowski, D. (1991): Middle Pleistocene 
stratigraphy of Poland: a review.- Proc. Geol. Assoc., 102: 201-215.

LindNer, L. (1995): Till sequences and local moraines in the Holy Cross Mountains area in central Poland. In: EHLERs, J. et al. [Hrsg.] Glacial Deposits in North-East Europe: 329-337; Rotterdam (Balkema).

Linke, G. \& Hallik, R. (1993): Die pollenanalytischen Ergebnisse der Bohrungen Hamburg-Dockenhuden (qho 4), Wedel (qho 2) und Hamburg-Billbrook.Geol. Jb., A 138: 169-184, 4 Abb., 1 Tab., 4 Taf.; Hannover.

Menke, B. (1968): Beiträge zur Biostratigraphie des Mittelpleistozäns in Norddeutschland (pollenanalytische Untersuchungen aus Westholstein). - Meyniana, 18: 35-42, 6 Abb.; Kiel.

Menke, B. (1975): Vegetationsgeschichte und Florenstratigraphie Nordwestdeutschlands im Pliozän und Frühquartär. - Mit einem Beitrag zur Biostratigraphie des WeichselFrühglazials. - Geol. Jb., A 26: 3-151, 9 Abb., 3 Tab., 8 Taf.; Hannover.

Meyer, K.-D. (1981): Arbeitsergebnisse der Subkommission für Europäische Quartärstratigraphie: Stratotypen des Elsterund Weichsel-Glazials. - Eiszeitalter u. Gegenwart, 31: 203-209; Hannover.

MeYer, K.-D. (1982): Erläuterungen zu Blatt 2524 Buxtehude. Geol. Karte Niedersachsen 1:25 000, Bl. 2524, 120 S., 22 Abb., 8 Tab., 7 Kt.; Hannover.

Mojski, J.E. (1995): Pleistocene glacial events in Poland. In: Ehlers, J. et al. [Hrsg.]: Glacial Deposits in North-East Europe: 287-292, 1 Abb., 1 Tab.; Rotterdam (Balkema).

MülLER, H. (1974): Pollenanalytische Untersuchungen und Jahresschichtenzählungen an der holsteinzeitlichen Kieselgur von Munster-Breloh. - Geol. Jb., A 21: $107-$ 140, 10 Abb., 2 Tab.; Hannover.

Müller, H. \& Höfle, H.-Chr. (1994): Die Holstein-Interglazialvorkommen bei Bossel westlich von Stade und Wanhöden nördlich Bremerhaven. - Geol. Jb., A 134: 71-116, 13 Abb., 5 Tab.; Hannover.

Overbeck, F. (1975): Botanisch-geologische Moorkunde unter besonderer Berücksichtigung der Moore Nordwestdeutschlands als Quellen zur Vegetations-, Klima- und Siedlungsgeschichte, 719 S., 263 Abb., 38 Tab.; Neumünster (Wachholtz).

Rzechowski, J. (1996): The Ferdynandowian Interglacial and its stratigraphical position in the Middle Pleistocene of Europe. In: Turner, Ch. [Hrsg.]: The early Middle Pleistocene in Europe: 279-317; Rotterdam, (Balkema).

Urban, B., Lenhard, R., Mania, D. \& Albrecht, B. (1991): Mittelpleistozän im Tagebau Schöningen, Ldkr. Helmstedt. - Z . dt. geol. Ges., 142: 351-372, 6 Abb., 2 Tab., 1 Taf.; Hannover.

West, R.G. (1980): The pre-glacial Pleistocene of the Norfolk and Suffolk coasts.- 203 S., 36 Taf.; Cambridge.

ZAGWIJN, W.H. (1975): Chronostratigrafie en biostratigrafie. Indeling van het Kwartair op grond van veranderingen in vegetatie en klimaat.- Toelichtingen Geolog. Overzichtskaarten van Nederland, 109-114; Haarlem.

ZAGwiJn, W.H. (1996): The Cromerian Complex Stage of the Netherlands and correlation with other areas in Europe. In: Turner, Ch. [ed.]: The early Middle Pleistocene in Europe: 145-172; Rotterdam (Balkema).

ZaGWIJn, W.H. \& DE Jong, J. (1984): Die Interglaziale von Bavel und Leerdam und ihre stratigraphische Stellung im niederländischen Früh-Pleistozän. - Meded. rijks geol. Dienst, 37: 155-169, 12 Abb., 6 Taf.; Maastricht.

ZaGWIJN, W.H. \& ZonNeveld (1956): The interglacial of Westerhoven.- Geol. Mijnbouw NS. 18, 37-46, 3 Abb. 